\title{
Comparing performance and robustness of SVM and ANN for fault diagnosis in a centrifugal pump
}

\author{
$\underline{\text { M. Saberi }}^{\text {a }}{ }^{\text {A. Azadeh }}{ }^{\text {b }}$, A. Nourmohammadzadeh ${ }^{b}$ and P. Pazhoheshfar ${ }^{c}$ \\ ${ }^{a}$ Institute for Digital Ecosystems \& Business Intelligence, Curtin University of Technology, Perth, Australia \\ ${ }^{b}$ Department of Industrial Engineering, Center of Excellence for Intelligent-Based Experimental Mechanics, \\ College of Engineering, University of Tehran, Iran \\ ${ }^{c}$ Islamic Azad University, Tafresh Branch, Young Researchers Club, Tafresh, Iran \\ Email: Mortezza_Saber@yahoo.com
}

\begin{abstract}
Fault detection and diagnosis has an effective role for the safe operation and long life of systems. Condition monitoring is an appropriate way of the maintenance techniques which is applicable in the fault diagnosis of rotating machinery faults. We considered the Support Vector Machine (SVM) method for classifying the condition of centrifugal pump into two types of faults through six features: flow, temperature, suction pressure, discharge pressure, velocity, and vibration. The SVM method is based on statistical learning theory (SLT) and powerful for the problem with small sampling, nonlinear and high dimension. (L.V. Ganyun et al 2005). The SVM classifying is implemented with 4 kernel functions and the results of them are compared. We use an Artificial Neural Network (ANN) as the second classifying method to have comparison among the performance of two methods. After applying the two methods to our data set we make the data set noisy and again we try our SVMs and ANN to compare their robustness in noisy conditions and the results obtained from two methods confirmed the superiority of SVM with some specific kernel functions.
\end{abstract}

Keywords: $\quad$ Support Vector Machine (SVM), Artificial Neural Network (ANN), Centrifugal pump, noisy condition 
Saberi et al., Comparing performance and robustness of SVM and ANN for fault diagnosis...

\section{INTRODUCTION}

Centrifugal pumps are sensitive to: (1) variations in liquid condition (i.e., viscosity, specific Gravity, and temperature); (2) suction variations, such as pressure and availability of a continuous volume of fluid; and (3) variations in demand. Several reasons cause mechanical failures Some are induced by cavitations, hydraulic instability, or other system-related problems. Others are the direct result of improper maintenance, maintenance-related problems, improper lubrication, misalignment, unbalance, seal leakage, and a variety of others that machine reliability is periodically affected by them. Inclusion of process parameters in a maintenance program can be accomplished in two ways:

manual or microprocessor-based systems. Both methods normally require installing instrumentation to measure the parameters that indicate the actual operating condition of plant systems. Even though most plants have installed pressure gauges, thermometers, and other instruments that should provide the information required for this type of program, many of them are no longer functioning. The pump is necessary for Public utilities and industry have been brought to a high state of technical development. Maintenance of mechanical equipment is generally distributed into four Species, describing different strategies towards maintenance. These species are as follow:

1. Breakdown/run-to-failure maintenance.

2. Preventive/time-based maintenance

3. Predictive/condition-based maintenance.

4. Pro-active/prevention maintenance

It is generally suggested for firms to implement an appropriate mix of these maintenance strategies to be cost-effective. There is a notion that the preventive methodologies and predictive maintenance methodologies involve high cost in terms of manpower and equipment to maintain machines. The major equipment failures in an oil and gas plant are related to pumps, compressors and piping. Pumps are amongst the oldest working machines used by man. The centrifugal pump already described 1689 in the "Acta Eruditorium" by the Marburg professor Denis Papin met with only limited use by the main customers at that time such as public water supply utilities. Condition monitoring of mechanical equipment is an accepted industrial practice to improve plant safety, efficiency and reliability. Condition monitoring is generally viewed as a predictive maintenance technique. Various diagnostics methods have been proposed for different types of rotating machinery. The task of condition monitoring and fault diagnosis of rotating machinery faults is both significant and important but is often the failure diagnosis process by human operators is time consuming and human error may lead to a faulty diagnosis(K. Rezaie 2010). Therefore two up-to-date and effective methods, Support Vector Machine (SVM) and an Artificial Neural Network (ANN) is used by this study to classify the condition of our system to 2 types of fault. Each of these two faults consists of some detailed faults.

(Karim Salahshoor et al 2010) presented a new FDD scheme for condition machinery of an industrial steam turbine using a data fusion. Fusion of a SVM machine with an ANFIS (adaptive neuro-fuzzy inference system) classifier, integrated into a common framework, is utilized to enhance the fault detection and diagnostic tasks. (CHANG Yan-wei 2008) proposed a new method based on principal component analysis (PCA) and SVMs for fault diagnosis of mine hoists. These results show that the algorithm using the RBF kernel function for the SVM had the best classification properties. (Qi Wu 2010) used robust wavelet Vsupport vector classifier machine (RWv-SVC) for complex fault diagnosis system. In article (LI Yunfen et al 2010), a nonlinear dynamic multiway partial least squares (MPLS) based on support vector machines (SVM) is developed for on-line fault detection in batch processes. The survey (Achmed Widodo and Bo-Suk Yang 2007) reviewed the use of condition monitorind and fault diagnosis with SVM method.

Accurate predictions from the ANN (Artificial neural network) models in combination with an undemanding integration in the power plant's computer system were some of the main conclusions from the study. The other survey used this approach is (V.G. Grishin et al 2003) that aims to describe the prospective methodology (Pictorial Analysis) of a human-computer interaction capable to select and adjust visual representations for better feature and pattern selection.

In following section we describe the SVM and ANN methods, apply the approaches to our data, analyze the results and we compare the robustness of these approaches in a noisy condition. In Table 1 we compared the methods and characteristics in other surveys in comparison with this study. 
Saberi et al., Comparing performance and robustness of SVM and ANN for fault diagnosis...

Table 1. Comparison between this study and others in this field

\begin{tabular}{|l|l|l|l|l|}
\hline \multicolumn{1}{|c|}{ Features } & SVM & ANN & FUZZY & $\begin{array}{l}\text { Other } \\
\text { methods }\end{array}$ \\
\hline Survey & $\sqrt{ }$ & & & \\
\hline Wang,M.H et al & & & & $\sqrt{ }$ \\
\hline Wu, Q. and Ni, Z. & $\sqrt{ }$ & & $\sqrt{ }$ & \\
\hline Yiakopoulos & & & & $\sqrt{ }$ \\
\hline L.V.Ganyun et al & $\sqrt{ }$ & & & \\
\hline CHANGYan-wei & $\sqrt{ }$ & & & $\sqrt{ }$ \\
\hline LI Yunfen et al & $\sqrt{ }$ & & & $\sqrt{ }$ \\
\hline Sheng-Fa Yuan & $\sqrt{ }$ & & & \\
\hline M.Fast and T.Palme & & $\sqrt{ }$ & \\
\hline V.G. Grishin et al & & $\sqrt{ }$ & \\
\hline K.Rezaie et al & & & $\sqrt{ }$ \\
\hline Kankar, P.K. et al & $\sqrt{ }$ & $\sqrt{ }$ & \\
\hline Achmed Widodo & $\sqrt{ }$ & & & \\
\hline Houxi Cui et al & $\sqrt{ }$ & & & $\sqrt{ }$ \\
\hline Du, S. and Xi, L. & & & & $\sqrt{ }$ \\
\hline K.Slahshoor & $\sqrt{ }$ & & & \\
\hline S.Mahadevan & $\sqrt{ }$ & & & \\
\hline LI Yunfen et al & $\sqrt{ }$ & & \\
\hline This study & & & & \\
\hline
\end{tabular}

As Table 1 shows in the most of surveys one of the methods SVM and ANN is applied and the only surveys, which used both approaches is (Kankar, P.K. et al 2011). In the mentioned papers the examination of methods in noisy condition is rarely implemented, while in this study we used the SVM and ANN, with making comparison between them in normal and noisy condition.

\section{REVIEW OF SVM}

Support vector machine was originally introduced by Vapnik 1998 and co-workers in the late 1990s. While traditional statistical theory keeps to empirical risk minimization (ERM), SVM satisfies structural risk minimization (SRM) based on statistical learning theory (SLT), whose decision rule could still obtain small error to independent test sampling. SVM mainly has two classes of applications, classification and regression. In this paper, application of classification is discussed.

This distinguishing function is the so-called SVM. From the above analysis, it can be concluded that SVM is decided by training samples and kernel function. The construction and selection of kernel function is important to SVM. But the kernel function is often given directly in practice. Some common kernel functions are shown as follows:

1. The polynomial kernel function has two forms as follows:

$$
\begin{aligned}
& \mathrm{K}(\mathrm{x}, \mathrm{y})=\langle\mathrm{x}, \mathrm{y}\rangle^{d} \\
& \mathrm{~K}(\mathrm{x}, \mathrm{y})=(\langle\mathrm{x}, \mathrm{y}\rangle+1)^{\mathrm{d}}
\end{aligned}
$$

2. Gaussian radial basis function is a universal kernel function

$\mathrm{K}(\mathrm{x}, \mathrm{y})=\exp (-|| x, y \| / 2 \sigma 2)$

3. B-splines are another popular formulation. The kernel is defined on the interval $[-1,1]$ and has an attractive closed form,

$\mathrm{K}(\mathrm{x}, \mathrm{y})=\mathrm{B}_{2 \mathrm{~N}+1}(\mathrm{x}-\mathrm{y})$ 
where $\mathrm{B}_{2 \mathrm{~N}+1}$ is the uniform $\mathrm{B}$-spline of $2 \mathrm{~N}+1$ order.

4. More complicated kernels can be obtained by summing and multiplying kernels,

$\mathrm{K}(\mathrm{x}, \mathrm{y})=\sum_{i} k_{i}(x, y) \quad \mathrm{K}(\mathrm{x}, \mathrm{y})=\prod_{i} k_{i}(x \cdot y)$

where $\mathrm{Ki}(\mathrm{x}, \mathrm{x})$ is a kernel function.

SVM has some potential advantages, which are listed below.

- It has clear concept, solid theory base and simple structure.

- It is a strongly regularized method, which is appropriate for ill-posed problems.

- It provides a unique solution and has a high training speed.

\section{ARTIFICIAL NEURAL NETWORK}

Artificial neural networks are a favorable alternative to econometric models. An ANN is an information processing paradigm that is inspired from the biological nervous systems, such as the brain processes information. ANNs, like people, have ability to learn by example. An ANN is configured for a specific application, such as pattern recognition, function approximation or data classification, through a learning process. Learning in biological systems involves adjustments to the synoptic connections that exist - among neurons. This is true for ANNs as well. They are made up of simple processing units which are linked by weighted connections to form structures that are able to learn relationships between sets of variables. This heuristic method can be useful for nonlinear processes that have unknown functional forms .

The feed forward neural networks or multi layer perceptron (MLP) among different networks are most commonly used in engineering. MLP networks are normally arranged in three layers of neurons, the input layer and output layer represent the input and output variables of the model, laid between them one or more hidden layers that hold the networks ability to learn non-linear relationships.

Architecture selection which is one major issue with implications on the empirical results consists of:

- Input and output variables number.

- Hidden layers' number.

- Hidden and output activation functions.

- Learning algorithm

All of the above issues are still open questions though there exist several answers to each one of them. The hidden units' number is determined by a trial-error process Few neurons in hidden layers (hidden units) can lead to under fitting, while too many neurons can cause over fitting. The actual number of neurons required in the hidden layer must be found by trial and error. Moreover, the inputs used by the network must be effective on the value of output(s), in fact the input and output variables should be identified carefully, because they enable the network to learn relationships faster and to use fewer hidden units . Another critical. Error in each neuron is the difference between ANN output and real output. The interconnecting weight and threshold value in each neuron is adjusted to minimize the error.

\section{METHODOLOGY}

We can summarize our methodology in steps as follows

Step1: Input determination : Choose the dimension of input variables or features through them condition of system or fault types are detected. We should pay attention to features which affect the condition of system or cause faults. Detection of effective feature is of great importance in this step.

Step2: Data division: Dividing the input data in two groups a train group to build our SVMs structure or train our artificial neural network and a test group to examine the performance of the SVMs or ANN. 
Saberi et al., Comparing performance and robustness of SVM and ANN for fault diagnosis...

It's better to consider a large number of rows(about 80\% ) for training group to have a good structure and use the rest as the test group.

Step3: Applying proposed methods: Apply the prepared SVM structure or trained ANN to our test group and get the outputs, which are classes of 1 or 2 for each row.

Step4: Choose the best parameters for methods : Implement the previous step with different initial parameters such as various kernel functions for SVM or some training methods for ANN

Step5: Comparing methods by their performance: Compare the classes determined by SVM or ANN with real classes to find the number of errors which determine the performance of the methods if we have only few errors our method works well.

Step6: Enter noise into data: Make noises in input data by adding a small value to about 40 percent of some columns or features

Step7: Apply methods to noisy data : Apply the SVMs and ANN to our new noisy data and get the output classes.

Step8: Choose best parameters for noisy data: compare the performances to determine the best kernel functions or trained methods

Step9: Comparing the robustness of methods in noisy condition: Compare the obtained performances using normal data (step2) and noisy data (step6) to determine which of methods has more robustness in noisy environment.

\section{RESULT AND ANALYSIS}

We applied above steps to our data as it is explained bellow.

Step1 for applying step 1 we used the data obtained from (K.Rezaie et al 2010) which detected the main features to monitor the condition of system. The determined feature are flow, temperature, suction pressure, discharge pressure, velocity, and vibration. We had 150 rows of data each of them has seven columns. The first six indicate different features of our centrifugal pump and the last one shows the class (1 or 2), which shows the fault type . Our main method for classifying in this survey is SVM because it has very appropriate in the case of having small sampling, nonlinear and high dimension.( L.V. Ganyun et al 2005).

Step2 We used 100 first rows for training our SVM structure and ANN. the next 50 rows for testing the performance of the structure.

Step3 We used 4 different kernel functions which are: linear, quadratic, gaussian and polynomial and compared the performance of them to determine which one works better. The second approach is artificial neural network which is used by numerous papers in the term of classifying and clustering. We have built a neural network with 3 layers with three and two three perceptrons in second and third layer and as we know an ANN classifier has percepron in its first layer equal to input variables.

Step4 The SVM method is started with the simplest kernel function which is linear .In many problems the linear approach has better performance in comparison with other complicated function .we continued with other kernel functions quadratic, gaussian and polynomial. The result we obtained with Matlab software is shown in Table 2.

Step5 In both of two approaches we have the classes, which are determined by classifier and the next step is to compare them with the real classes to realize the performance of the method and choose the best one.

Step6 The other aim to use two methods is to compare the robustness of them in a noisy environment . Hence we make our data sets noisy by adding the value 0.1 to 40 percent of column 1 and 6 . 
Saberi et al., Comparing performance and robustness of SVM and ANN for fault diagnosis...

Step7 and 8 The result in Table 2 shows that SVM method has an appropriate performance in problems like this Among 4 kernel functions Gaussian has the best performance and then is linear but we may like to choose linear because it's the simplest. Also we can find that the SVM approach is very robust in noisy environment because the differences in performance rate is very small. The gaussian and linear function have the largest robustness and no differences is existed between normal and noisy environments. It should be noticed that in some unlikely cases such as this case with quadratic function noise causes better performance rate.

Step9 As Table 3 shows, the number of error increases and become two times larger but the ANN is also robust against noise. But in comparison between two methods if we apply gaussian or linear kernel functions the SVM seems to be more powerful because its performance is larger, totally robust to face noises and more simple to apply .

At the end of this section, we show an integrated result of all methods including performance in normal condition, performance in noisy condition and the differences between them, which indicates robustness of our algorithm.

\section{CONCLUSION}

The scope of this study was find the effective and robust approach to control the condition of centrifugal pump to detect two kind of fault. We had 150 rows of data each of them has 6 dimensions which are the features. We used the first 100 rows for building the SVM structures either training ANN and the next 50 rows for testing the methods. The SVMs method with Gausian and linear function have superiority because of larger performance, robustness in noisy environments and also simplicity.

Table2. Result obtained by SVM the correct rate and number of errors by different kernel functions

\begin{tabular}{|c|c|c|c|c|c|}
\hline & Functions & Linear & Quadratic & Gaussian & polynomial \\
\hline \multirow{2}{*}{$\begin{array}{l}\text { Correct rate and } \\
\text { number of errors in } \\
50 \text { examples }\end{array}$} & Normal data & $\begin{array}{c}0.96 \\
2\end{array}$ & $\begin{array}{c}0.88 \\
6\end{array}$ & $\begin{array}{l}1 \\
0\end{array}$ & $\begin{array}{c}0.86 \\
7\end{array}$ \\
\hline & Noisy data & $\begin{array}{c}0.96 \\
2\end{array}$ & $\begin{array}{c}0.92 \\
4\end{array}$ & $\begin{array}{l}1 \\
0\end{array}$ & $\begin{array}{c}0.84 \\
8\end{array}$ \\
\hline
\end{tabular}

Table 3 .ANN classifying result

\begin{tabular}{|c|c|c|c|c|c|}
\cline { 2 - 6 } & & Linear & Quadratic & Gaussian & polynomial \\
\hline \multirow{2}{*}{$\begin{array}{c}\text { Correct rate and } \\
\text { number of errors in } \\
50 \text { examples }\end{array}$} & Normal data & 0.96 & 0.88 & 1 & 0.86 \\
\cline { 2 - 6 } & Noisy data & 0.96 & 6 & 1 & 7 \\
& & 2 & 4 & 0.92 & 0.84 \\
\end{tabular}

Table 4. The integrated result of all methods

\begin{tabular}{|c|l|l|l|l|}
\cline { 2 - 5 } \multicolumn{1}{c|}{} & & Normal & noisy & difference \\
\hline \multirow{3}{*}{ SVM } & Linear & 0.96 & 0.96 & 0 \\
\cline { 2 - 6 } & Quadratic & 0.88 & 0.92 & -0.04 \\
\cline { 2 - 6 } & Gaussian & 1 & 1 & 0 \\
\cline { 2 - 5 } & polynomial & 0.86 & 0.84 & 0.02 \\
\hline
\end{tabular}


Saberi et al., Comparing performance and robustness of SVM and ANN for fault diagnosis...

\section{REFERENCES}

A User's Guide to Support Vector Machines, Asa Ben-Hur, Jason Weston NEC Labs America

Achmad Widodo, Bo-Suk Yang ,Support vector machine in machine condition monitoring and fault diagnosis, Mechanical Systems and Signal Processing 21 (2007) 2560-2574

C.Yan-wei,W.Yao-cai,L.Tao,W.Zhi-jie, Fault diagnosis of a mine hoist using PCA and SVM techniques, J China Univ Mining \& Technol 18 (2008) 0327-033118 (2008) 0327-0331

Chang, C.-T., Chen, C.Y. Fault diagnosis with automata generated languages. 2011

diagnosis of a combined heat and power plant, Energy 35 (2010) 1114-1120

Du, S., Xi, L. Fault diagnosis in assembly processes based on engineering-driven rules and PSOSAEN algorithm 2011

K.Rezaie,V.Ebrahimpour, and S.Nouri, A fuzzy approach to improve fault diagnostic system in centrifugal pumps, 2010

K.Slahshoor,M.Kordestani,M.S.Khoshro, Fault detection and diagnosis of an industrial steam turbine using fusion of SVM (support vector machine) and ANFIS(adaptive nero-fuzzy inference system) classifiers, Energy 35 (2010) 5472e5482

Kankar, P.K., Sharma, S.C., Harsha, S.P. Fault diagnosis of ball bearings using machine learning methods 2011.

L. Yunfen, W.Zhifeng, Y. Jingqi, On-line Fault Detection Using SVM-based Dynamic MPLS for Batch Processes, Chinese J. Chem. Eng., 14(6) 754-758 (2006)

L.V. Ganyun, Cheng Haozhong, Zhai Haibao, Dong Lixin, Fault diagnosis of power transformer based on multi-layer SVM classifier, Electric Power Systems Research 74 (2005) 1-7

Qi Wu, Car assembly line fault diagnosis based on robust wavelet SVC and PSO, Expert Systems with Applications 37 (2010) 5423-5429

S.Deng, Seng-Yi Lin, We-Luan Cheng Application of multiclass support vector machines for fault 2011

S.Mahadevan, S.L. Shah, Fault detection and diagnosis in process data using one-class support

V.G. Grishin, Pictorial AnalysiA. Abraham, Rule-based expert systems. In: P.H. Sydenham and -R. Thorn, Editors, Handbook of measuring system design, John Wiley \& Sons, Ltd. 2005, Article 130.s of Experimental Data (in Russian), Nauka Publishing, vector machines, Journal of Process Control 19 (2009) 1627-1639

V.Vapnik, statistical learninig theory ,John Wesley and Songd ,1998

Wang.M.H,chung.Y.K,Sung.W.T Using thermal image matter-element to design a circuit board fault diagnosis system 2011

$\mathrm{Wu}, \mathrm{Q}$. Hybrid fuzzy support vector classifier machine and modified genetic algorithm for automatic car assembly fault diagnosis 2011

Wu, Q., Ni, Z. Car assembly line fault diagnosis based on triangular fuzzy Gaussian support vector classifier machine and modified genetic algorithm 2011

Yiakopoulos, C.T., Gryllias, K.C., Antoniadis, I.A. Rolling element bearing fault detection in industrial environments based on a K-means clustering approach2011 